\title{
The role of digital technology in bridging the child mental health care gap
}

\author{
Daniel Fatori ${ }^{1} \cdot$ Guilherme V. Polanczyk ${ }^{1}$
}

Published online: 19 March 2019

○) Springer-Verlag GmbH Germany, part of Springer Nature 2019

The field of child psychiatry has come a long way in the last decades. There is now a wide range of effective interventions available to treat child mental disorders, from pharmacotherapy and psychotherapy to complex psychosocial programs [1]. However, these interventions are probably not accessible for most of children with mental disorders worldwide [2]. The unmet need is known to be related to policy gaps (e.g., lack of specific policies for child mental health) and implementation gaps (e.g., insufficient number of mental health professionals). Epidemiological studies focused on describing unmet needs in children across the globe are crucial to understand the access gap and to provide information to plan health systems. Once there is a clear picture of the unmet needs, more resources are expected to be directed to the field, which is considerably under-funded [3]. More importantly, monitoring changes in rates of unmet needs over long periods of time can provide much-needed evidence on the effectiveness of strategies used to reduce the child mental health care gap.

The study by Lempinen et al. [4] is an important contribution to the field, showing positive changes in rates of mental health service use by children across a 24 -year period. The authors reported on a time-trend study comprised of four cross-sectional studies in Finland (986 children in 1989, 891 in 1999, 930 in 2005, and 942 in 2013) with high rates of participation (86-95\%). Parents and teachers reported on children's conduct, emotional, and hyperactivity symptoms using the Rutter A2 and B2 questionnaires, as well as if they considered that the child needed mental health treatment or if they actually sought mental health treatment for their child. Reports of mental health service use increased from 2.4 to $11.0 \%$ between 1989 and 2013. Moreover, among children with high levels of symptoms in at least

Daniel Fatori

daniel.fatori@gmail.com

1 Department of Psychiatry, Faculdade de Medicina FMUSP, Universidade de Sao Paulo, São Paulo, SP, Brazil one domain, mental health service use increased from 7.6\% in 1989 to $32.7 \%$ in 2013. Among those in most need (high level of symptoms in two or three domains), the increase was even more pronounced, from $18.3 \%$ in 1989 to $50.7 \%$ in 2013. The authors also sought to investigate if family characteristics explain differences in the reports of mental health service use. The full multivariate multinomial logistic regression model, adjusted by year and child sex, showed that living in a non-nuclear family and reporting symptoms of conduct disorders, emotional disorders, and hyperactivity predicted considered and reported use of mental health service use.

The remarkable increase in Finland on the reported mental health service use rate among children in need can be explained by important and gradual changes that happened over 2 decades in the country, such as policy changes and increases in financial and human resources. Other countries must learn the lessons from Finland on how to tackle the issue. Nevertheless, there is the other side of the coin: in 2013 , half of the children with high levels of symptoms did not receive treatment in this country. Furthermore, the quality, duration, and efficacy of the treatment received were not measured, making it difficult to understand if adequate treatment to specific needs was being delivered. Even a developed country with a well-organized health system, with a high human development index, and with a high number of child psychiatrists has difficulties in closing the gap. Figures are dramatic in developing countries: in the most developed cities of Brazil, $81 \%$ of the children diagnosed with at least one mental disorder did not receive treatment [5]. Data are lacking in most of the country, especially in the poorest areas, which is true for the majority of the developing world, where $90 \%$ of the worldwide population of children live.

How can the lack of data be addressed? Epidemiological surveys are complex and expensive studies, limiting their implementation across the world. Moreover, interviews and retrospective questionnaires are time-consuming, limited by memory bias and lack of ecological validity, potentially 
leading to inaccurate data. Digital technology can play an important role in circumventing these issues. The growing ubiquity of smartphones has facilitated the implementation of real-time data collection via mobile apps, resulting in a growing interest in studies using this methodology in child psychiatry in the last years $[6,7]$. Smartphone apps can easily be programmed to prompt participants to answer questions about behaviors or symptoms, as well as mental health services use in specific times of the day throughout any period of time. Health facilities in a given study area can be mapped and the respective GPS locations can trigger questions about treatment if participants are nearby. As a consequence, trajectories of service use across time and space can be identified dynamically in real time. It is a promising strategy that can advance the field in understanding the trajectories of services use and quality, duration, and satisfaction of treatments received.

In addition, mobile technology can be integrated into community health care, helping overcome one of the most important barriers preventing children from getting access to treatment: low levels of detection of mental disorders [8]. However, a few studies tested the use of mobile technology in this context [9]. The next wave of studies needs to go beyond the use of conventional scales and use the much strength of statistical algorithms. In this regard, computerized adaptive testing, commonly used in the field of education, is a promising tool. Computerized adaptive testing is a computer-administered assessment in which the sequences of items are determined by the previous responses, resulting in a dynamic test able to reduce a 1-h-long interview to less than 5 min [10]. Promising work using this tool to diagnose depression and anxiety in adults in the primary care system has been done [11]. In the future, due to its standardized and structured nature, computerized adaptive tests developed to detect common mental disorders in children can easily be administered by community health workers. Considering that most countries do not have specialized professionals to diagnose children in need [12], the high cost of training child psychiatrists, and the low use of services, community health workers equipped with mobile devices with computerized adaptive tests could provide support in detecting and referring children with mental disorders.

Bridging the child mental health care gap will be one of the most difficult and crucial challenges of the next decade [13]. Innovative strategies using digital technology are already transforming the field of child psychiatry [14]. However, digital technology has an immense potential that has yet to be fulfilled. Future studies should use real-time passive and active data collection to better understand trajectories and aspects related to services use, and test the efficacy of statistical algorithms, such as computerized adaptive testing, in detecting children with mental disorders in the community.
Funding DF is funded by Grant 2016/13451-9 São Paulo Research Foundation (FAPESP). GVP is funded by Grant 2016/22455-8, São Paulo Research Foundation (FAPESP) and Grant 310582/2017-2, and National Council for Scientific and Technological Development (CNPq).

\section{References}

1. Kieling C, Baker-Henningham H, Belfer M et al (2011) Child and adolescent mental health worldwide: evidence for action. Lancet 378:1515-1525

2. Rocha TB-M, Graeff-Martins AS, Kieling C, Rohde LA (2015) Provision of mental healthcare for children and adolescents: a worldwide view. Curr Opin Psychiatry 28:330-335. https://doi. org/10.1097/YCO.0000000000000169

3. Pollitt A, Cochrane G, Kirtley A, Krapels J, Larivière V, Lichten CA, Parks S, Wooding S (2016) Project ecosystem: mapping the global mental health research funding system. RAND Corporation, Santa Monica. https://www.rand.org/pubs/research_reports/ RR1271.html

4. Lempinen L, Luntamo T, Sourander A (2018) Changes in mental health service use among 8-year-old children: a 24-year time-trend study. Eur Child Adolesc Psychiatry. https://doi.org/10.1007/ s00787-018-1218-9

5. Fatori D, Salum GA, Rohde LA et al (2019) Use of mental health services by children with mental disorders in two major cities in Brazil. Psychiatr Serv. https://doi.org/10.1176/appi.ps.201800389

6. Baltasar-Tello I, Miguélez-Fernández C, Peñuelas-Calvo I, Carballo JJ (2018) Ecological momentary assessment and mood disorders in children and adolescents: a systematic review. Curr Psychiatry Rep 20:66

7. Miguelez-Fernandez C, de Leon SJ, Baltasar-Tello I et al (2018) Evaluating attention-deficit/hyperactivity disorder using ecological momentary assessment: a systematic review. Atten Defic Hyperact Disord. https://doi.org/10.1007/s12402-018-0261-1

8. Patel V, Kieling C, Maulik PK, Divan G (2013) Improving access to care for children with mental disorders: a global perspective. Arch Dis Child 98:323-327

9. Naslund JA, Aschbrenner KA, Araya R et al (2017) Digital technology for treating and preventing mental disorders in low-income and middle-income countries: a narrative review of the literature. Lancet Psychiatry 4:486-500

10. Gibbons RD, Weiss DJ, Frank E, Kupfer D (2016) Computerized adaptive diagnosis and testing of mental health disorders. Annu Rev Clin Psychol 12:83-104

11. Graham AK, Minc A, Staab E et al (2019) Validation of the computerized adaptive test for mental health in primary care. Ann Fam Med 17:23-30

12. Bruckner TA, Scheffler RM, Shen G et al (2011) The mental health workforce gap in low- and middle-income countries: a needs-based approach. Bull World Health Organ 89:184-194

13. Patel V, Saxena S, Lund C et al (2018) The Lancet Commission on global mental health and sustainable development. Lancet. https ://doi.org/10.1016/S0140-6736(18)31612-X

14. Powell L, Parker J, Harpin V (2018) What is the level of evidence for the use of currently available technologies in facilitating the self-management of difficulties associated with ADHD in children and young people? A systematic review. Eur Child Adolesc Psychiatry 27:1391-1412 